\title{
La Educación Entre Pares Como Estrategia Para Prevenir Accidentes de Tránsito en Motociclistas de la Facultad de Ciencias Para la Salud de la Universidad de Caldas
}

\author{
${ }^{1}$ Helberth Augusto González-Rico, ${ }^{2}$ Bryan S Urrea, ${ }^{3}$ Fabián E Puentes-Manosalva
}

\begin{abstract}
RESUMEN
Introducción y objetivos: En Manizales los motociclistas han resultado los mayores contraventores de normas de tránsito, viéndose involucrados en 5 de cada 10 accidentes y responsables de casi el $50 \%$ de los muertos en choques. Con el proyecto 'enciende tu vida' buscamos concientizar a la población en torno a esta problemática y prevenir los accidentes que involucran jóvenes motociclistas, en primer lugar en la población universitaria de la Facultad de Ciencias para la Salud de la Universidad de Caldas, usando la educación entre pares como principal estrategia, generando en ellos empoderamiento como agentes multiplicadores.
\end{abstract}

Materiales y métodos: Se llevaron a cabo actividades de sensibilización y educación a cerca de 150 motociclistas, divididas en tres etapas que incluían impacto audiovisual, autoevaluación y reconocimiento de conductas al conducir motocicleta, educación entre pares en torno a las características del buen y mal motociclista, así como conocimiento de señales de tránsito. Todas las actividades se apoyaron de folletos, calcomanías, manillas, encuestas, y cubrimiento periodístico. Los datos fueron tabulados y procesados con Epilnfo ${ }^{\mathrm{TM}}$. Se implementará esta estrategia en otras ciudades y facultades del país a través de los distintos capítulos de la Asociación Colombiana Médica Estudiantil.

Resultados: El $71,2 \%$ de los motociclistas encuestados, consideraba que tenía prácticas inadecuadas al conducir. Así mismo, el $80,3 \%$ reconoció que la educación a motociclistas es la mejor estrategia para prevenir accidentes de tránsito, considerando que desde que se implementó la educación entre pares dentro del campus, el desempeño de la mayoría de ellos

\footnotetext{
${ }^{1}$ Student (Sixth Year), ${ }^{2}$ Student (3rd Year)

${ }^{3}$ General Surgeon and Gastroenterologist

1,2Departamento Clínico - Quirúrgico, Programa de Medicina Facultad de Ciencias para la Salud, Universidad de Caldas Manizales, Colombia; Asociación Colombiana Médica Estudiantil (ACOME)

${ }^{3}$ Departamento Clínico - Quirúrgico, Programa de Medicina Facultad de Ciencias para la Salud, Universidad de Caldas Manizales; Unión de Cirujanos Manizales, Colombia

Corresponding Author: Helberth Augusto González-Rico Student (Sixth Year), Departamento Clínico - Quirúrgico Programa de Medicina, Facultad de Ciencias para la Salud Universidad de Caldas, Manizales, Colombia, Phone: $(+57)$ 31151366 47, e-mail: helberth38@hotmail.com
}

$(72,7 \%)$ será más seguro, estando en capacidad de transmitir recomendaciones a otros motociclistas.

Conclusiones: La Educación entre pares es una estrategia útil y efectiva en motociclistas universitarios al hablar de seguridad vial, bajo la premisa de que éstos serán más propensos a escuchar y responder positivamente a la información suministrada cuando viene de sus pares, como lo sería otro estudiante universitario.

Palabras claves: Accidentes, Educación entre pares, Motocicletas, Prevención, Tráfico.

How to cite this article: González-Rico HA, Urrea BS, PuentesManosalva FE. La Educación Entre Pares Como Estrategia Para Prevenir Accidentes de Tránsito en Motociclistas de la Facultad de Ciencias Para la Salud de la Universidad de Caldas. Panam J Trauma Crit Care Emerg Surg 2015;4(3): 155-164.

Source of support: Nil

Conflict of interest: None

\section{ABSTRACT}

Introduction and objectives: In Manizales, motorcyclists have been the biggest violators of traffic rules and are involved in 5 out of 10 accidents and account for almost $50 \%$ of those killed in clashes. The project 'turns your life' seeks to raise public awareness about this problem and prevent accidents involving young motorcyclists, first in the student population of the Faculty of Health Sciences at the University of Caldas, using peer education as a primary strategy, creating empowering them as multipliers.

Materials and methods: Awareness raising and education were done in about 150 riders, divided into three stages, including visual impact, self-assessment and recognition of behaviors when driving motorcycle, peer education about the characteristics of good and evil biker and knowledge of traffic signals. All activities are supported by leaflets, stickers, bracelets, surveys, and journalistic coverage. Data were processed and tabulated with Epi Info ${ }^{\mathrm{TM}}$. This strategy will be implemented in other cities of the country through the various chapters of the Colombian Medical Student Association.

Results: Around $71.2 \%$ of respondent motorcyclists considered to have inadequate driving practices. Also, $80.3 \%$ said that education of motorcyclists is the best strategy to prevent traffic crashes, Since peer education campus was implemented, the performance of most of them $(72.7 \%)$ was safer, being able to convey recommendations to other motorcyclists.

Paper presented in Student Research Competition in PTS Congress, Panama City, Panama, Nov 2014. 
Conclusion: Peer education is a useful and effective strategy in university motorcyclists to talk about road safety, under the premise that they will be more likely to listen and respond positively to the information provided when it comes from their peers, as would other college students.

Keywords: Accidents, Motorcycle, Peer education, Prevention, Traffic.

\section{INTRODUCCIÓN}

Si bien, la mayoría de los estudiantes ven en la medicina preventiva una herramienta útil para el ejercicio profesional, ello no necesariamente expresa una adecuada actitud, o satisfacción al realizar dichas actividades, ${ }^{1}$ sobre todo en sistemas de salud como el nuestro en donde la medicina curativa tiene un valor superior. Sin embargo, desde la Asociación Colombiana Médica Estudiantil (ACOME), una organización autónoma, sin ánimo de lucro y políticamente independiente que vela por la formación integral de los estudiantes de medicina colombianos, buscamos situar al estudiante de medicina en una realidad social donde la falta de impacto de programas de prevención de accidentes de tránsito y seguridad vial, nos atañe. De ahí que buscando hacer frente a esta problemática surja un proyecto educativo, iniciativa de un grupo de estudiantes preocupados por los altos índices de accidentalidad particularmente en jóvenes motociclistas de nuestra región, usando como principal estrategia la educación entre pares.

Al referirnos a educación entre pares, hablamos de un enfoque en la que miembros de la comunidad reciben apoyo para promover el cambio que mejora la salud entre personas en su misma condición. La educación entre pares es la enseñanza o el intercambio de información sobre la salud, los valores y el comportamiento en la educación de otras personas que pueden compartir similares orígenes sociales o experiencias de la vida. ${ }^{2} \mathrm{~A}$ través de un proceso de participación, la educación entre pares crea un entorno en el que las personas se sienten seguras y capaces de compartir información, valores, habilidades, etc. En general los programas de ayuda entre iguales han producido influencias positivas en los centros educativos, desarrollando en los estudiantes una mayor educación emocional y potenciando la reflexión sobre el impacto de sus acciones en los demás, ${ }^{3}$ lo cual es uno de los objetivos que se persigue con la implementación de un programa educativo de esta índole.

En general la mayoría de programas de educación entre pares se han centrado en temas de salud sexual y reproductiva, pues resulta ser un tema de interés entre adolescentes y adultos jóvenes. Sin embargo, según datos del Observatorio regional en salud de la OPS los accidentes de tránsito representan la segunda causa de mortalidad dentro de personas entre 20 y 25 años, ${ }^{4}$ por lo que un programa de educación entre pares relacionado con este tema se justifica en este grupo etario. La educación entre pares también se ha utilizado en proyectos de salud que pretenden reducir la incidencia del habito de fumar, el consumo de alcohol o el abuso de sustancias psicoactivas en los jóvenes. ${ }^{5}$ De ahí que implementar un programa de educación entre pares relacionado con la prevención de accidentes de tránsito en motociclistas resulte de utilidad y conveniencia. El objetivo general del proyecto es educar a jóvenes motociclistas en medidas de prevención de accidentes de tránsito y generando a su vez en ellos empoderamiento como agentes multiplicadores entre sus pares, ayudando a cambiar las actitudes frente a la seguridad en las vías, sensibilizándolos sobre la problemática, a fin de evitar prácticas inadecuadas al momento de conducir.

\section{ANTECEDENTES}

La Organización Mundial de la Salud (OMS) en sus estudios ha comprobado que la incidencia y la mortalidad generada por los accidentes de tránsito son comparables con el de otras enfermedades como la producida por el VIH. Se ha calculado que 50 millones de personas en el mundo anualmente resultan afectadas por los accidentes de tránsito y se calcula incluso que aumentara hasta en un $80 \%$ en los países en vía de desarrollo durante los próximos años. ${ }^{6}$

Cada año se producen en todo el mundo aproximadamente 1,24 millones de muertes por accidentes de tránsito, y la situación ha cambiado poco desde 2007. Sin embargo, esta estabilización debe examinarse en el contexto de un aumento mundial del 15\% en el número de vehículos registrados, asociado a la facilidad en la adquisición de los mismos, lo cual indica que las intervenciones para mejorar la seguridad vial mundial han mitigado en cierta medida el aumento previsto del número de muertes. ${ }^{7}$ Este alto número de muertes ha llevado a los accidentes de tránsito a posicionarse como la octava causa de muerte en el mundo y pese a que los países en el mundo cada vez mejoran en las estrategias de promoción y prevención el número sigue en aumento y se prevé que de no tomarse medidas más drásticas al año 2030 será la quinta causa de muerte en el mundo.

A nivel nacional durante los últimos años la tendencia se ha incrementado según lo demuestran las estadísticas del Instituto de Medicina Legal y Ciencias Forenses del año 2009 que muestran que la mortalidad por accidentes de tránsito en Colombia se ubica en el segundo lugar de las causas de muerte violenta con un porcentaje aproximado del $22 \% .{ }^{8}$ Más de la mitad de las personas que mueren en el mundo por accidentes de tránsito están representadas 
entre peatones, ciclistas y motociclistas, sin embargo las cifras varían entre las regiones y entre los países entre otras cosas por el número de motocicletas que circulan en las diferentes zonas. Al comparar con otros países del mundo se encuentra que Colombia presenta un $42 \%$ de siniestros en los cuales se encuentran involucradas motocicletas, este valor en comparación con otros países desarrollados y en vía de desarrollo es el valor más alto. ${ }^{9}$ Aproximándonos a la epidemiologia local y según datos suministrados por la Secretaria de Tránsito y Transporte de Manizales, por cada por cada 10 lesionados en accidentes de tránsito: 5 iban en moto, 3 eran peatones y 2 usaban otro medio de transporte, lo cual deja ver la problemática y el grupo frente al cual deben centrarse los esfuerzos. Según informes del Fondo de Prevención Vial, apoyados por cifras del Instituto Nacional de Medicina Legal, en Manizales para el año 2010 hubo 13 muertos y 367 heridos en siniestros que involucraban motocicletas, para el 2011 se documentó 14 muertos y 440 heridos y para el 2012 se registraron 18 muertos y 516 heridos, lo cual muestra el ascenso progresivo en la mortalidad relacionada con accidentes en motocicleta. ${ }^{10}$ De igual manera vale la pena resaltar que del total de conductores infractores el $17.88 \%$ conducía motocicleta sin observar las normas de tránsito (6.613 infracciones).

La Organización Panamericana de la Salud (OPS) realizó un estudio entre 2006 y 2007 centrándose en seguridad vial, en el que Colombia se sitúa en la cuarta posición en muertes por accidente de tránsito con 5490 muertes. De igual manera se encontró que los mayores factores de riesgo en Latinoamérica son el NO uso del casco en los motociclistas, el NO uso del cinturón de seguridad en automóviles y el conducir bajo los efectos del alcohol. En relación a esto de gran preocupación el NO usar casco por parte de los motociclistas ya que en nuestro país es el factor de riesgo de mayor presentación por encima del $60 \%$ y se encuentra entre los mayores de Latinoamérica de la mano con Suriname, Cuba, Chile y Canadá. ${ }^{11}$

Ahora bien en cuanto a los rangos de edad más afectados en Colombia, al igual que la estadística global, se ha registrado que los más vulnerables son las personas menores de 30 años, tanto mujeres como hombres, aunque con mayor mortalidad representada en estos últimos. Hacia el año 2010 mueren en Colombia 2.044 personas por accidentes de tránsito dentro de este rango de edad. De estas se registró que la mayor cantidad de muertes de presentan en personas entre los 20 y 25 años y en su mayor parte se asocia al uso de motocicleta como medio de transporte. De igual manera se comporta el número de personas lesionadas con la diferencia evidente que las cifras son mucho más altas, y la mayoría de ellos sufre lesiones que muy posiblemente afecte su calidad de vida futura.

\section{MATERIALES Y MÉTODOS}

Buscando implementar un programa educativo dirigido a la comunidad universitaria se creó un proyecto denominado Enciende tu vida, con el objetivo de direccionarlo desde la ACOME. Se llevaron a cabo actividades de sensibilización y educación a cerca de 150 motociclistas de la Facultad de Ciencias para la Salud de la Universidad de Caldas, divididas en tres etapas, en tres días diferentes del mes de Abril de 2014. La primera de ellas consistió en impacto audiovisual mediante la proyección de vídeos e imágenes al ingreso en los que los motociclistas podían observar conductas inadecuadas y consecuencias de las mismas, a la vez que se empezaba a difundir el logotipo creado para el proyecto (Figura 1), en el marco de una campaña de expectativa. Un segundo momento buscó en enfrentar a los motociclistas a sus conductas al conducir mediante la aplicación de una autoevaluación. Finalmente, un tercer momento incluyó intervención educativa entre pares (Figura 2), en las que miembros de ACOME orientaron sobre las características del buen y mal motociclista a través de un decálogo creado para tal fin, así como conocimiento de señales de tránsito, apoyados por folletos, calcomanías y manillas, bajo la premisa de que un estudiante universitario estará más propenso a responder positivamente a la información que suministra alguien en su misma condición, distinto a si ésta proviniera de una autoridad (por ejemplo, un agente de tránsito). Se recogió una muestra evaluativa de las actividades desarrolladas, buscando retroalimentar y enriquecer el proyecto para posteriormente implementarlo en otras facultades del país.

El impacto de un proyecto de intervención de estas características resulta atractivo para organismos del sector publico dada las características de la población a la que va dirigido, pues según informes del Fondo de Prevención Vial las principales víctimas fatales

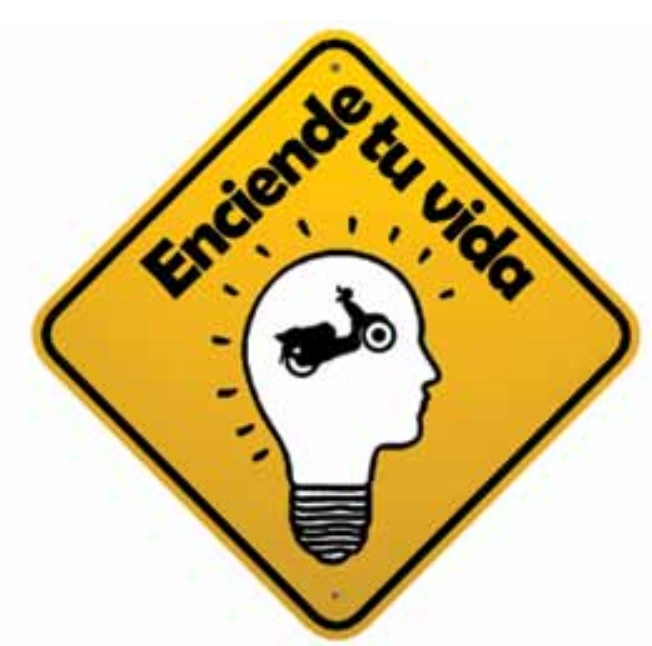

Figura 1: Logotipo del proyecto 


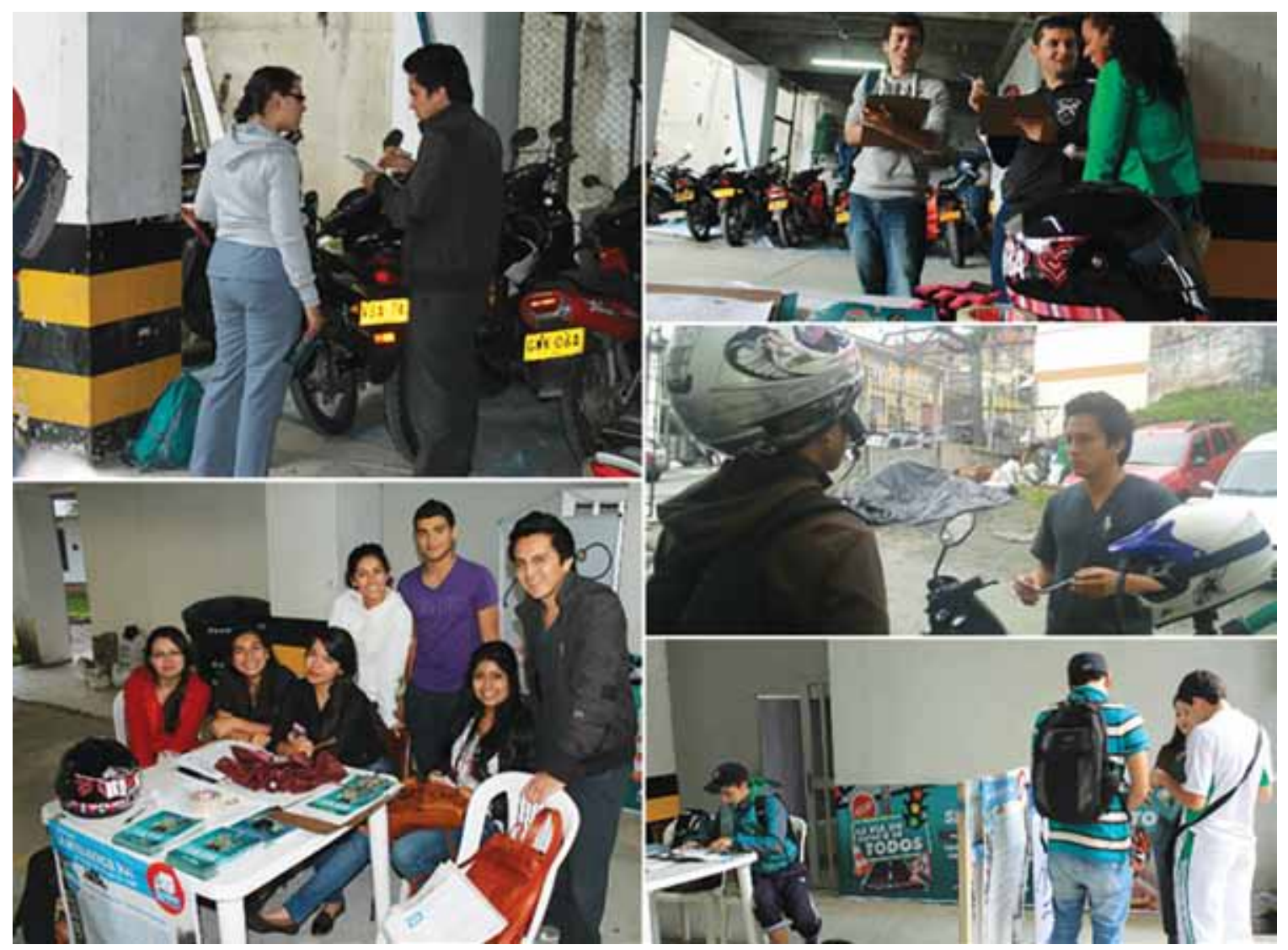

Figura 2: Actividad educativa implementada basada en la educación entre pares

involucradas en accidente de tránsito en motocicleta tanto para las mujeres como para los hombres son los jóvenes, en el rango de 20-29 años, ${ }^{9}$ y como tal es un proyecto que se desarrolló primordialmente en este grupo etario. Por tal motivo se logró apoyo de la Secretaria de Tránsito y Transporte de Manizales, incluso integrando las actividades a la semana de la Educación Vial. De igual manera buscando enriquecer y divulgar esta iniciativa, se contó con el cubrimiento periodístico del medio local de mayor difusión. Los datos recogidos en las actividades fueron tabulados y procesados con EpiInfo ${ }^{\mathrm{TM}}$.

\section{RESULTADOS}

Se recolectó un total de 149 encuestas dentro de estudiantes que participaron de las actividades educativas, 83 de autoevaluación de conductas al conducir motocicleta (Tabla 1) y 66 de evaluación del impacto y utilidad de las actividades desarrolladas (Tabla 2). Se impactó sobre la población (Tabla 3) que según estadística nacional ${ }^{9}$ representa la mayor mortalidad de accidentes de tránsito en motocicleta, género masculino entre 20 y 30 años (75,9\%). La mayoría de los participantes $(71,2 \%)$ reconoció haber tenido conductas inadecuadas al conducir motocicleta, con cambios importantes en la percepción de éstas tras la implementación del programa educativo, lo que orienta hacia cambios positivos y conductas más seguras al conducir. De igual forma, la mayoría de los participantes $(80,3 \%)$ cree que la educación es la estrategia de mayor utilidad en la prevención de accidentes de tránsito en motocicleta, calificando como efectiva la educación entre pares. Si bien hay conductas que muchos tienen claramente identificadas como adecuadas, como adelantar a otro vehículo por izquierda $(85,5 \%)$ el programa reforzó este tipo de conductas encontrando que tras la intervención el 92,4\% de los participantes sabía por dónde debía adelantar. Dentro de las conductas incorrectas de muchos de los participantes, sobresale el adelantar en medio de dos vehículos $(67,5 \%)$ lo cual es una práctica común e insegura de los motociclistas, así como la realización de giros indebidos $(58,2 \%)$ por tratarse de un vehículo pequeño.

\section{DISCUSIÓN}

Como tal el objetivo principal del proyecto no es hacer una caracterización epidemiológica de la población intervenida respecto a la accidentalidad en motocicleta, sino evaluar el impacto y receptividad de un programa educativo entre pares, pudiendo encontrar resultados alentadores, pues centrando la intervención en un decálogo de conductas adecuadas e inadecuadas muchas de las personas que participaron se sensibilizaron frente a esta problemática, identificaron actitudes que muchas veces tienen al conducir y la mayoría $(77,2 \%)$ se siente en la capacidad de replicar el mensaje a compañeros, 
Tabla 1: Autoevaluación de conductas al conducir motocicleta (previa implementación del programa educativo)

\begin{tabular}{|c|c|c|}
\hline Ítem valorado (pregunta) & $n$ & $\%$ \\
\hline Tiempo de conducción de motocicleta & 83 & \\
\hline Menos de 6 meses & 6 & $(7.23)$ \\
\hline Menos de 1 año & 9 & $(10.84)$ \\
\hline Entre 1 y 5 años & 34 & $(40.96)$ \\
\hline Más de 5 años & 34 & $(40.96)$ \\
\hline $\begin{array}{l}\text { Frecuencia con la que realiza mantenimiento } \\
\text { a la motocicleta }\end{array}$ & 83 & \\
\hline Cada mes & 11 & $(13.25)$ \\
\hline Entre 2 y 3 meses & 23 & $(27.71)$ \\
\hline Entre 4 y 6 meses & 18 & $(21.69)$ \\
\hline Entre 7 y 11 meses & 8 & $(9.64)$ \\
\hline Cada año & 21 & $(25.30)$ \\
\hline Nunca & 21 & $(2.41)$ \\
\hline Motocicleta acorde con estatura & 83 & \\
\hline $\mathrm{Si}$ & 68 & $(81.93)$ \\
\hline No & 15 & $(18.07)$ \\
\hline $\begin{array}{l}\text { Haber realizado modificaciones a la } \\
\text { motocicleta }\end{array}$ & 83 & \\
\hline $\mathrm{Si}$ & 26 & $(31.33)$ \\
\hline No & 57 & $(68.67)$ \\
\hline $\begin{array}{l}\text { Modificaciones realizadas están autorizadas } \\
\text { por la ley }\end{array}$ & 26 & \\
\hline $\mathrm{Si}$ & 17 & $(65.38)$ \\
\hline No & 2 & $(7.69)$ \\
\hline No sabe & 7 & $(26.92)$ \\
\hline $\begin{array}{l}\text { Contar con elementos de seguridad } \\
\text { obligatorios }\end{array}$ & 83 & \\
\hline $\mathrm{Si}$ & 79 & $(95.18)$ \\
\hline No & 4 & $(4.82)$ \\
\hline Adelantar entre dos vehículos & 83 & \\
\hline $\mathrm{Si}$ & 56 & $(67.47)$ \\
\hline No & 27 & $(32.53)$ \\
\hline $\begin{array}{l}\text { Uso de direccionales para girar o cambiar } \\
\text { de carril }\end{array}$ & 83 & \\
\hline Siempre & 66 & $(79.52)$ \\
\hline Casi siempre & 13 & $(15.66)$ \\
\hline Pocas veces & 4 & $(4.82)$ \\
\hline Acostumbra a adelantar un vehículo por: & 83 & \\
\hline Derecha & 3 & $(3.61)$ \\
\hline Izquierda & 71 & $(85.54)$ \\
\hline Le es indiferente & 9 & $(10.84)$ \\
\hline Acostumbra a manejar & 82 & \\
\hline Rápido (alta velocidad) & 17 & $(20.73)$ \\
\hline Despacio (poca velocidad) & 7 & $(8.54)$ \\
\hline Velocidad intermedia & 58 & $(70.73)$ \\
\hline Uso de cebras como atajos & 82 & \\
\hline $\mathrm{Si}$ & 14 & $(17.07)$ \\
\hline No & 68 & (82.93) \\
\hline Realización de giros indebidos & 79 & \\
\hline $\mathrm{Si}$ & 46 & $(58.23)$ \\
\hline No & 33 & $(41.77)$ \\
\hline Haber conducido bajo efectos del alcohol & 83 & \\
\hline $\mathrm{Si}$ & 18 & $(21.69)$ \\
\hline No & 65 & $(78.31)$ \\
\hline Haber pasado semáforos en rojo & 83 & \\
\hline $\mathrm{Si}$ & 36 & $(43.37)$ \\
\hline No & 47 & $(56.63)$ \\
\hline
\end{tabular}

Tabla 2: Resultados de encuesta de salida (impacto y utilidad de las actividades desarrolladas)

\begin{tabular}{lll}
\hline Ítem valorado (pregunta) & $n$ & $\%$ \\
\hline $\begin{array}{l}\text { Percepción respecto a la educación entre } \\
\text { pares: }\end{array}$ & 66 & \\
$\quad$ Efectiva & 64 & $(96,97)$ \\
$\quad$ Inefectiva & 2 & $(3,03)$ \\
Sentirse en capacidad de transmitir & 66 &
\end{tabular}

recomendaciones a otros motociclistas, familiares y amigos

$\begin{array}{lll}\mathrm{Si} & 51 & (77,27) \\ \mathrm{No} & 1 & (1,52) \\ \text { Intermedio } & 14 & (21,21) \\ \begin{array}{l}\text { Autopercepción respecto a la presencia de } \\ \text { prácticas inadecuadas a la hora de conducir } \\ \text { motocicleta }\end{array} & 66 & \\ \mathrm{Si} & & \\ \mathrm{No} & 47 & (71,21) \\ \text { Cambio en la percepción de prácticas } & 19 & (28,79)\end{array}$

inadecuadas después de la implementación del programa

$\begin{array}{lll}\text { Si, tuvo impacto } & 63 & (95,45) \\ \text { No, continúo opinando lo mismo } & 3 & (4,55)\end{array}$

Percepción del desempeño al conducir moto 66 tras la implementación del programa

$\begin{array}{lll}\text { Más seguro } & 48 & (72,73) \\ \text { Igual de seguro } & 18 & (27,27) \\ \text { Menos seguro } & 0 & (0)\end{array}$

Tras implementar el programa al adelantar $\quad 66$ un vehículo lo hará por:

$\begin{array}{lll}\text { Derecha } & 3 & (4,55) \\ \text { Izquierda } & 61 & (92,42) \\ \text { Le es indiferente } & 2 & (3,04)\end{array}$

Percepción respecto a la mejor estrategia $\quad 66$ para prevenir accidentes de tránsito en motocicleta

Más educación

Mejor señalización

Mayor presencia de agentes de tránsito

Penas más fuertes a infractores 5

Conocimiento de las consecuencias de $\quad 66$ cometer una infracción

\begin{tabular}{cll}
$\mathrm{Si}$ & 64 & $(96,97)$ \\
$\mathrm{No}$ & 2 & $(3,03)$ \\
Volver a cometer una infracción de tránsito/ & 66 & \\
conducta inadecuada al conducir motocicleta & & \\
$\mathrm{Si}$ & 7 & $(10,61)$ \\
$\mathrm{No}$ & 59 & $(89,39)$ \\
\hline
\end{tabular}

amigos, familiares, etc., lo que representa un paso importante en la construcción de agentes multiplicadores de cambio. La formación es un paso importante para la prevención, ya que se pasa del conocimiento a la concienciación. Esto se traduce en que cada uno de quienes se involucraron en el proyecto toma consciencia de los riesgos y responsabilidades que comporta conducir una motocicleta.

Con la implementación de este programa ratificamos que cada escenario de la cotidianidad se puede convertir 
Tabla 3: Características generales de la población intervenida

\begin{tabular}{lll}
\hline & $n$ & $\%$ \\
\hline Edad (<20 años) & 83 & \\
$<20$ & 6 & $(7,22)$ \\
$20-24$ & 42 & $(50,6)$ \\
$25-29$ & 21 & $(25,3)$ \\
$30-34$ & 12 & $(14,4)$ \\
35 o más & 2 & $(2,4)$ \\
Genero & 83 & \\
Masculino & 53 & $(63.84)$ \\
Femenino & 30 & $(36.14)$ \\
Programa académico & 83 & \\
Educación física & 24 & $(28.92)$ \\
Medicina & 20 & $(24.10)$ \\
Enfermería & 14 & $(16.87)$ \\
Regencia en farmacia & 5 & $(6.02)$ \\
Otro & 20 & $(24.10)$ \\
\hline
\end{tabular}

en una oportunidad de impartir educación a nuestros pares, en temas de índole general como lo es la educación vial. El objetivo a mediano y largo plazo es implementar esta estrategia en otras ciudades y facultades del país, tras convertirla en proyecto de intervención nacional de la ACOME, desarrollándolo en los distintos capítulos, partiendo del precepto de que los motociclistas serán más propensos a responder positivamente a la información que suministran sus pares, en este caso otro estudiante universitario.

Como estudiantes de medicina y como miembros del gremio de la salud tenemos una responsabilidad adicional frente a conductas preventivas de esta índole. Los estudiantes de medicina ingresan a la universidad con creencias, concepciones y valores propios que influirán en sus prácticas posteriores en el campo médico y personal. Generalmente estas percepciones se correlacionan con el comportamiento individual. ${ }^{12}$ De ahí la importancia de fortalecer las actitudes positivas y tratar de aminorar o eliminar aquellas negativas mediante un mejor y mayor conocimiento de aspectos preventivos en escenarios como el de la educación vial, pues si bien no constituye un tema médico-científico, constituye un tema al que nos enfrentamos con relativa frecuencia.

Con el avance de la tecnología, los métodos de ayuda entre iguales también tienen en cuenta las posibilidades de ayuda a distancia, ${ }^{3}$ como lo puede ser el uso de internet, redes sociales, e-mail, etc., lo cual constituyo una herramienta útil en la difusión del proyecto, así como el apoyo que se consiguió por medio de la prensa local, pues mientras más llegada a la comunidad se tuvo, más se pudo conocer el proyecto y por lo tanto, más posibilidades de participación y beneficio a la población a la que iba dirigido.

Tras la implementación del programa, se concluye que la educación entre pares es una estrategia útil y efectiva en motociclistas universitarios al hablar de seguridad vial, bajo la premisa de que éstos serán más propensos a escuchar y responder positivamente a la información suministrada cuando viene de sus pares. Este enfoque fue aprovechado no sólo como forma de intervención sino como una instancia de participación y crecimiento personal, pues cada uno de los participantes exploró habilidades comunicativas y de interacción, en la medida que se convertían agentes de cambio. A través de la educación entre pares se comparten experiencias (adecuadas o inadecuadas), y por lo tanto se está en mejor posición para proveer información relevante, significativa, explícita y sincera.

\section{REFERÊNCIAS}

1. García JC. Enseñanza de la medicina preventiva y social. La educación Médica en la América Latina. Washington DC: Organización Mundial de la Salud. Publicación Científica 1972;255:86-167.

2. Green J. Peer education. Promotion \& Education 2001 June;8: 65-68.

3. Cartwright N. Setting up and sustaining peer support systems in a range of schools over 20 years. Pastoral Care 2005;23:45-50.

4. Sistema de información Regional de Mortalidad. Observatorio Regional de Salud. Organización Panamericana de Salud. Recurso interactivo disponible online http://ais.paho.org/ phip/viz/rho_sa_causas_principales_mortalidad_es.asp.

5. ONU. Droga y el delito. Utilización de las estrategias de comunicación interpares para prevenir el uso indebido de drogas, Nueva York; ONU; 2003.

6. Instituto Nacional de Salud Subdirección de Vigilancia y Control en Salud Pública - SIVIGILA. Boletín Epidemiológico Semanal, Semana epidemiológica Boletín epidemiológico, Ministerio de la Protección Social de Colombia, Dirección General de Salud Pública, Bogotá DC 2004;17.

7. Organización Mundial de la Salud - OMS. Informe sobre la situación mundial de la seguridad vial 2013, Apoyo al decenio de acción. Organización Mundial de la Salud, Departamento de Prevención de la Violencia y los Traumatismos y Discapacidad. Ginebra, Suiza: Organización Mundial de la Salud 2013.

8. Corporación Fondo de la Prevención Vial. Anuario Estadístico de Accidentalidad vial en Colombia. Bogotá D.C: UNIANDES 2010.

9. Corporación Fondo de Prevención Vial. Anuario estadístico de accidentalidad vial en Colombia 2011. Uniandes, Grupo de estudios en sostenibilidad urbana y regional-Sur. Bogotá: Corporación Fondo de Prevención Vial 2013.

10. Fondo de Prevención Vial. (Julio de 2012). Corporación Fondo de Prevención Vial. (Dd.-C. Vial, Editor, \& Instituto Nacional de Medicina Legal) Recurso interactivo disponible en http:// www.fpv.org.co/investigacion/estadisticas.

11. Organización Panamericana de la Salud. Informe sobre el estado de la seguridad vial en la región de las Américas. Organización Panamericana de la Salud, Washington 2009.

12. Garr D. Prevention education and evaluation in US Medical schools: A status report. Acad Med 2000;75(7):14-21. 


\section{La Educación Entre Pares Como Estrategia Para Prevenir Accidentes de Tránsito en Motociclistas de la Facultad de Ciencias Para la Salud de la Universidad de Caldas}

A nivel mundial fallecen anualmente 5.8 millones de personas por trauma, de ellas casi 1.300 .000 mueren por lesiones ligadas al tránsito y entre 20 y 50 millones de personas sufren consecuencias no mortales por esta causa. Las lesiones por este mecanismo son la principal causa de muerte entre 5 y 44 años y dentro de las muertes provocadas por el tránsito, los motociclistas representan un $23 \%$. Estas últimas cifras se incrementan hasta $30 \%$ en países de medianos y bajos ingresos. ${ }^{1}$

Este problema es especialmente importante en América Latina, donde la cantidad de motociclistas lesionados por tránsito se incrementó en virtud de la expansión del uso de este tipo de vehículos en las dos últimas décadas. En Brasil, por ejemplo, la flota de motocicletas se incrementó 14 veces entre los años 1990 y 2008. ${ }^{2,3}$ El trabajo de González Rico y colaboradores ${ }^{4}$ publicado en este número de la Revista Panamericana de Trauma, Cuidado Crítico y Cirugía de Emergencia resulta de especial interés por la elección del rango etario de 20-29 años como objetivo de la intervención, dado que es esta franja en la cual ocurre la mayor parte de los siniestros viales. ${ }^{5}$

Las estrategias guiadas por pares, modelo de intervención elegido por estos autores, constituyen un abordaje viable y económico que ha sido utilizado en diversos escenarios: pacientes con enfermedades mentales, ${ }^{6}$ cáncer $^{7}$ y estimulación de la lactancia materna ${ }^{8,9}$ con resultados variables. Una de las críticas que más frecuentemente se realiza a este tipo de intervenciones es que su desarrollo no se inscribe en un proceso de mejora de indicadores específicos, producto de lo cual es muy difícil evaluar su efectividad. Por otro lado, dado lo heterogéneo de estas intervenciones, resulta imposible encontrar una herramienta única que pueda aplicarse de forma universal más allá del objetivo específico que persigue cada una de ellas. Más aún, cuando estas estrategias fueron evaluadas en ensayos clínicos randomizados, la heterogeneidad de los estudios impidió la extracción de conclusiones sólidas.

En el artículo comentado, 150 estudiantes fueron incluidos en una estrategia de trabajo que estuvo conformada por: (1) Proyección de videos con conductas inadecuadas de conducción; (2) Aplicación de una autoevaluación sobre conductas al conducir; (3) Intervención educativa entre pares: algunos miembros de la Asociación Colombiana Médica Estudiantil (ACOME) orientaron sobre características de buen y mal motociclista mediante un decálogo diseñado por los autores para este fin, seguida de una segunda autoevaluación sobre el impacto de esa estrategia. La proporción de respuestas obtenidas fue 55\% para la instancia de autoevaluación (83 de 150) y de $44 \%$ (66 de 150) para la evaluación de impacto. En base a esto, los autores consideraron la intervención como positiva al haber logrado una mejoría en aspectos específicos, como la identificación de pautas de conducción adecuadas. Es de considerar que con estas tasas de respuesta la población estudiada puede no ser representativa del total de la muestra evaluada.

Uno de los aspectos llamativos de la encuesta de autoevaluación es que más del 60\% informó realizar mantenimiento de su vehículo cada 6 meses o más frecuentemente, lo cual habla de un nivel previo de concientización sobre seguridad vial bastante alto. Por otra parte, es llamativo que los autores no hayan incluido preguntas sobre el uso de casco en las encuestas.

Una de las principales conclusiones de los investigadores es que la educación de seguridad vial entre pares se revela como una estrategia útil y efectiva en motociclistas universitarios, considerando que serán más propensos a escuchar y responder positivamente a la información cuando ésta viene de sus pares. Si bien desde el punto de vista teórico esta afirmación es válida, no encontramos elementos suficientes en la investigación que logren sostenerla, por lo cual opinamos que esta conclusión debería ser relativizada.

Consideramos que utilizar una muestra más grande, mejorar los métodos para asegurar una mayor proporción de respuestas y aplicar una nueva medición mediante autoencuesta anónima a un período más alejado (i.e., 3, 6 o 12 meses post intervención) podría ayudar a sostener con más respaldo las conclusiones vertidas en este artículo.

Más allá de la metodología de este estudio, es poco lo que puede decirse sobre cambios reales en hábitos saludables. La mayor parte de estas intervenciones requieren sostenerse en el tiempo hasta poder apreciarse un cambio de conducta que repercuta sobre la integridad de la sociedad (en este caso, menos siniestros viales con víctimas).

Entendemos que esta iniciativa de González Rico y colaboradores es un buen paso en ese sentido y estamos de acuerdo con ellos en que el ámbito de los estudiantes de medicina es ideal para desarrollar actividades de prevención guiadas por pares. A su vez, entendemos que esta opción, asociada a una metodología de investigación sólida, 
sustentable y permanente será una herramienta de gran utilidad para prevenir las muertes y las secuelas de los siniestros viales en la población de 20 a 29 años de edad. Felicitamos a los autores por el compromiso con la educación y prevención demostrado durante este interesante estudio relacionado con el comportamiento del motociclista.

\section{REFERENCIAS}

1. Organización Mundial de la Salud. Plan Mundial para el Decenio de Acción para la Seguridad Vial 2011-2020.

2. Darido G. Concerns over the growth of motorcycles in cities. World Bank 2010. Disponible en: http://blogs.worldbank.org/ transport/concerns-over-the-growth-of-motorcycles-in-cities.

3. Estupiñan N, Santana M, Palacios A, Rodríguez DA. Motorcycle ownership and use: the case of Latin America. CAF-Development Bank of Latin America. Disponible en: https://www.academia.edu/3856834/Motorcycle_ownership_and_use_The_case_of_Latin_ America_Nicolas_Estupinan_et_al.

4. González Rico HA, Urrea Trochez BS, Puentes Manosalva FE. La Educación entre pares como estrategia para prevenir accidentes de tránsito en motociclistas de la Facultad de Ciencias para la Salud de la Universidad de Caldas. Colocar cita de acuerdo a la paginación final de la revista.

5. World Health Organization. Injuries and violence 2014: the facts. Disponible en: http://www.who.int/violence_injury_prevention/ media/news/2015/Injury_violence_facts_2014/en/.

6. Lloyd-Evans B, Mayo-Wilson E, Harrison B, Istead H, Brown E, Pilling S, Johnson S, Kendall T. A systematic review and metaanalysis of randomised controlled trials of peer support for people with severe mental illness. BMC Psychiatry 2014;14:39.

7. Macvean ML, White VM, Sanson-Fisher R. One-to-one volunteer support programs for people with cancer: A review of the literature. Patient Education and Counseling 2008;70:10-24.

8. Kaunonen M, Hannula L, Tarkka MT. A systematic review of peer support interventions for breastfeeding. J Clin Nurs 2012 Jul; 21(13-14):1943-1954.

9. Jolly K, Ingram L, Khan KS, Deeks JJ, Freemantle N, MacArthur C. Systematic review of peer support for breastfeeding continuation: metaregression analysis of the effect of setting intensity, and timing. BMJ 2012;344:d8287.

Jorge Neira MD FCCM FAAST

Presidente, Fundación Trauma Argentina

Ezequiel Monteverde

Director Registro de Trauma, Fundación Trauma Argentina 


\section{INVITED COMMENTARY}

\section{Peer Education as a Strategy to prevent Traffic Accidents in Motorcyclists of School of Health Sciences at the University of Caldas}

Worldwide, more than 5 million people die each year as a result of several injuries. Around 1.3 million are related to road traffic collisions, and about 20 to 50 million suffer from nonlethal consequences. Road traffic injuries are the main cause of death among people between 5 and 44 years old, $23 \%$ of whom are motorcycle riders. This rate rises up to $30 \%$ in low and middle-income countries. ${ }^{1}$

This problem is especially applicable to Latin America, where the rate of traffic-injured motorcyclists has grown increasingly over the last two decades, mainly due to the increasing use of this form of transportation. For example, motorcycle fleet in Brazil increased 14 times between 1990 and 2008. ${ }^{2,3}$ The article by González Rico et al ${ }^{4}$ published in this issue of PJTCCES is of special interest because of their election of students from 20 to 29 years old as the intervention group, the age range in which most of road traffic injuries occur. ${ }^{5}$

Peer-guided interventions are economically viable strategies that have been used in several different scenarios, in patients with mental ${ }^{6}$ and oncologic ${ }^{7}$ illnesses or in breastfeeding support ${ }^{8,9}$ with variable results. One of the most common criticisms comes from the fact that these approaches constitute a package of heterogeneous interventions with no specific performance indicators. When analyzed in randomized controlled trials, variation inherent to outcomes assessed avoided reaching reliable conclusions. The current evidence does not provide endorsement for the effectiveness of peer support interventions.

In this article by González Rico et al, 150 students were exposed to an intervention strategy composed by (1) the projection of an educational video on safe driving behavior, (2) a first self-evaluation on driving behavior, (3) a peerguided intervention: a group of members from the Colombian Medical Student Association (ACOME) interacted with the study population based on a Decalogue specifically designed for this purpose. This intervention was followed by a second self-evaluation regarding peer support intervention impact. The researchers informed a retrieval rate of $55 \%$ (83 out of 150) for the first survey and 44\% (66 out of 150) for the second one. They reported positive variations in some specific aspects, as an improvement in the identification of safe driving tips. However, it must be considered that with such a low retrieval rate, results may not properly reflect the characteristics of the entire sample.

One of the issues in the manuscript that called our attention was that more than $60 \%$ of the students reported being aware of their motorcycle maintenance needs and the fact that they kept them updated every 6 months or even more frequently. We interpret this conduct as a high previous level of awareness on this issue. Nevertheless we found no mention related to helmet use, neither in the first nor in the second self-evaluations. We recognize that helmet use is an important aspect to be considered given that, as the authors remind of in the Background section, it is the most important risk factor for traffic-related injuries in motorcyclists.

The main conclusion of this investigation is that peer-supported education on traffic safety reveals as a useful and effective strategy in undergraduate students riding motorcycles. This has the implicit observation that undergraduate students would be prone to better considering this kind of advice when coming from a peer rather than from an authority. Being this affirmation theoretically valid, we did not find enough elements in this investigation to support it, so we suggest considering it with caution.

In our opinion, we suggest the authors should work with a bigger sample, with the aim of improving the retrieval rate and adding a new anonymous self-evaluation at 3, 6 or 12 months from the intervention time, so it could lead to a better support of the assumptions made.

Beyond this study, little can be said regarding changes in driving habits in real life. These interventions must be long-term sustained until an attitudinal change could be perceived in the community, hoping it will lead to decreasing the amount of victims of motorcycle crashes. We salute this initiative carried out by González Rico et al. As a positive step toward developing better driving habits, we agree that medical students are an ideal group for this type of interventions.

We consider that this option, while associated to a solid, sustainable and permanent research methodology, might be a useful tool to prevent deaths and sequels from motor-vehicle crashes among the population between 20 and 29 years old and we congratulate the authors on the commitment to education and prevention showed during this interesting study related to motorcyclist behavior. 


\section{REFERENCES}

1. Organización Mundial de la Salud. Plan Mundial para el Decenio de Acción para la Seguridad Vial 2011-2020.

2. Darido G. Concerns over the growth of motorcycles in cities. World Bank 2010. Disponible en: http://blogs.worldbank.org/ transport/concerns-over-the-growth-of-motorcycles-in-cities.

3. Estupiñan N, Santana M, Palacios A, Rodríguez DA. Motorcycle ownership and use: the case of Latin America. CAF-Development Bank of Latin America. Disponible en: https://www.academia.edu/3856834/Motorcycle_ownership_and_use_The_case_of_Latin_ America_Nicolas_Estupinan_et_al.

4. González Rico HA, Urrea Trochez BS, Puentes Manosalva FE. La Educación entre pares como estrategia para prevenir accidentes de tránsito en motociclistas de la Facultad de Ciencias para la Salud de la Universidad de Caldas. Colocar cita de acuerdo a la paginación final de la revista.

5. World Health Organization. Injuries and violence 2014: the facts. Disponible en: http://www.who.int/violence_injury_prevention/ media/news/2015/Injury_violence_facts_2014/en/.

6. Lloyd-Evans B, Mayo-Wilson E, Harrison B, Istead H, Brown E, Pilling S, Johnson S, Kendall T. A systematic review and metaanalysis of randomised controlled trials of peer support for people with severe mental illness. BMC Psychiatry 2014;14:39.

7. Macvean ML, White VM, Sanson-Fisher R. One-to-one volunteer support programs for people with cancer: a review of the literature. Patient Education and Counseling 2008;70:10-24.

8. Kaunonen M, Hannula L, Tarkka MT. A systematic review of peer support interventions for breastfeeding. J Clin Nurs 2012 Jul; 21(13-14):1943-1954.

9. Jolly K, Ingram L, Khan KS, Deeks JJ, Freemantle N, MacArthur C. Systematic review of peer support for breastfeeding continuation: metaregression analysis of the effect of setting, intensity, and timing. BMJ 2012;344:d8287.

Jorge Neira MD FCCM FAAST

Presidente, Fundación Trauma Argentina

Ezequiel Monteverde

Director Registro de Trauma, Fundación Trauma Argentina 